\title{
Electron scattering from a mesoscopic disk in Rashba system
}

\author{
Jr-Yu Yeh and Ming-Che Chang* \\ Department of Physics, National Taiwan Normal University, Taipei, Taiwan \\ Chung-Yu Mou \\ Department of Physics, National Tsing Hwa University, Hsinchu, Taiwan
}

(Dated: May 12, 2018)

\begin{abstract}
Electrons with spin-orbit coupling moving in mesoscopic structures can often exhibit local spin polarization. In this paper, we study the influence of the Rashba coupling on the scattering of two-dimensional electrons from a circular disk. It is observed that spin-polarized regions exist, even if the incident electrons are unpolarized. In addition to the distributions of charge and spin current in the near-field region, we also analyze the symmetry and the differential cross-section of the scattering.
\end{abstract}




\section{INTRODUCTION}

Spin-orbit interaction influences the electronic and transport properties of semiconductors. For example, it lifts the degeneracy of the valence bands, modifies the electron g-factor, $\frac{1}{}$ and causes skew scattering in the presence of (spinless) impurities. Such a skew scattering is a possible mechanism for the extrinsic spin Hall effect. $\stackrel{2}{2}$ In addition, spin-orbit interaction plays an important role in the recently proposed intrinsic spin Hall effect $\underline{\underline{3} . \underline{4}}$ It is also crucial in the mechanisms of spin relaxation and optical orientation in semiconductors. $\underline{\underline{5}} \underline{\underline{6}}$

It is highly desirable to generate flows of polarized spins in semiconductors with the help of the spin-orbit interaction. In these endeavors, the Rashba spin-orbit coupling ${ }^{7}$ in two-dimensional electron gas (2DEG) plays a special role since it allows manipulation of spin flows by varying the gate bias. This has motivated several creative proposals for its application. ${ }^{-}$To explore the possibilities, the effect of Rashba coupling in many types of mesoscopic structure have been investigated, such as a quantum wire, $\frac{9}{9}$ a quantum ring, $\frac{10}{\underline{9}}$ and a quantum dot.11,12 In several studies, it was found that a device with a simple geometry, in combination with the Rashba coupling, could serve as a spin filter. For example, the device could be a T-shaped channel,,$\frac{13}{}$ a quantum point contact, $\stackrel{14}{ }$ parallel interfaces that cause double refraction, $\stackrel{15}{\underline{15}}$ or even just a curved wire $\stackrel{16}{\underline{6}}$ After applying a magnetic field, we could further build a spin filter based on electron focusing, $\frac{17}{,}$ or based on the an interferometer of the Stern-Gerlach type. 18

In this paper, we study the scattering of electrons by a disk in the 2DEG with Rashba coupling. ${ }^{19}$ The analysis can be applied to a wide range of situations in which the radius $R$ of the disk can be much smaller, roughly the same, or much larger than the electron wave length $\lambda$. For example, the usual impurity scattering can be simulated with $R \ll \lambda$, while the scattering by an artificial mesoscopic disk corresponds to $R \approx \lambda$. Here we focus on the latter case to search for possible effect of local spin generation. We find that, because of the Rashba coupling, spin-up and spin-down electrons are indeed separated and accumulate in regions of curved stripes. The associated charge and spin currents are analyzed in details. In addition, the properties of symmetry, as well as the differential cross-section, are also investigated..$^{20}$

This paper is organized as follows: Sec. II is the theoretical analysis. In Sec. III, the results from numerical calculations are presented. Sec. IV is the conclusion. 


\section{ANALYSIS OF THE DISK SCATTERING}

\section{A. Hard disk scattering}

Consider a two-dimensional electron system with a circular disk at the origin,

$$
H=\frac{p^{2}}{2 m}+\frac{\alpha}{\hbar}\left(\sigma_{x} p_{y}-\sigma_{y} p_{x}\right)+V(r)
$$

where $V(r)=V_{0}$ when $r \leq R$ and 0 otherwise. In the following, the potential of the disk is considered infinite ("hard" disk). The two-component wave function $\left(\psi_{1}, \psi_{2}\right)^{T}$ with energy $E$ satisfies the coupled equations:

$$
\begin{aligned}
& \left(\frac{\partial^{2}}{\partial r^{2}}+\frac{1}{r} \frac{\partial}{\partial r}+\frac{1}{r^{2}} \frac{\partial^{2}}{\partial \phi^{2}}+\frac{2 m E}{\hbar^{2}}\right) \psi_{1}+\frac{2 i m \alpha}{\hbar^{2}} e^{-i \phi}\left(i \frac{\partial}{\partial r}+\frac{1}{r} \frac{\partial}{\partial \phi}\right) \psi_{2}=0 \\
& \left(\frac{\partial^{2}}{\partial r^{2}}+\frac{1}{r} \frac{\partial}{\partial r}+\frac{1}{r^{2}} \frac{\partial^{2}}{\partial \phi^{2}}+\frac{2 m E}{\hbar^{2}}\right) \psi_{2}+\frac{2 i m \alpha}{\hbar^{2}} e^{i \phi}\left(-i \frac{\partial}{\partial r}+\frac{1}{r} \frac{\partial}{\partial \phi}\right) \psi_{1}=0 .
\end{aligned}
$$

It follows that the energy eigenstates of the Hamiltonian with momentum $k$, helicity $\eta$ $\left(E=\hbar^{2} k^{2} / 2 m+\eta \alpha k, \eta= \pm\right)$, and angular momentum $(n+1 / 2) \hbar$ (where $n$ is an integer) are

$$
\psi_{\eta n}(r, \phi)=\left(\begin{array}{c}
\Omega_{\eta n}(k r) e^{i n \phi} \\
\Omega_{\eta(n+1)}(k r) e^{i(n+1) \phi}
\end{array}\right),
$$

where $\Omega_{\eta n}$ can be a Bessel function, a Neumann function, or their linear combination, such as a Hankel function. We will choose the Hankel functions as the eigen-basis since their behavior at large radius suits the boundary condition for scattering.

The energy eigenstate of the Schroedinger equation with energy $E$ and angular momentum $(n+1 / 2) \hbar$ can be written as

$$
\begin{aligned}
\Psi_{n} & =a_{n}\left(\begin{array}{c}
H_{n}^{1}(k r) e^{i n \phi} \\
H_{n+1}^{1}(k r) e^{i(n+1) \phi}
\end{array}\right)+b_{n}\left(\begin{array}{c}
H_{n}^{2}(k r) e^{i n \phi} \\
H_{n+1}^{2}(k r) e^{i(n+1) \phi}
\end{array}\right) \\
& +c_{n}\left(\begin{array}{c}
H_{n}^{1}\left(k^{\prime} r\right) e^{i n \phi} \\
-H_{n+1}^{1}\left(k^{\prime} r\right) e^{i(n+1) \phi}
\end{array}\right)+d_{n}\left(\begin{array}{c}
H_{n}^{2}\left(k^{\prime} r\right) e^{i n \phi} \\
-H_{n+1}^{2}\left(k^{\prime} r\right) e^{i(n+1) \phi}
\end{array}\right)
\end{aligned}
$$

where $E=\hbar^{2} k^{2} / 2 m+\alpha k=\hbar^{2} k^{\prime 2} / 2 m-\alpha k^{\prime}$. The first two terms have positive helicity; while the other two terms have negative helicity. The most general eigenstate of the Schroedinger equation with energy $E$ is a superposition of the $\Psi_{n}$ 's, where the coefficients $a_{n}, b_{n}, c_{n}$, and $d_{n}$ are determined by boundary conditions. Because of the circular symmetry of the potential, 
the angular momentum is conserved for each $n$-component. Therefore, each component can be considered independent during the scattering.

The incident plane wave with momentum $k$ and helicity $\eta$ can be decomposed as the following linear superposition:

$$
\Psi_{\mathrm{in}, \eta}=e^{i k x} \frac{1}{\sqrt{2}}\left(\begin{array}{c}
1 \\
-\eta i
\end{array}\right)=\frac{1}{\sqrt{2}} \sum_{n=-\infty}^{\infty}\left(\begin{array}{c}
i^{n} J_{n}(k r) e^{i n \phi} \\
\eta i^{n} J_{n+1}(k r) e^{i(n+1) \phi}
\end{array}\right)
$$

The Bessel functions $J_{n}(k r)$ can be further decomposed as Hankel functions $H_{n}^{1}(k r)$ and $H_{n}^{2}(k r)$, the former correspond to outgoing circular waves, while the later correspond to incoming circular waves (with no phase shift). If an incident wave has a definite helicity, $\Psi_{\text {in,+ }}$, then by comparing Eq. (4) with the components of Eq. (5) at large distance, one will

obtain $b_{n}=i^{n} / 2 \sqrt{2}$ and $d_{n}=0$ (i.e. no incoming circular wave with negative helicity) for all $n$. The coefficients $a_{n}$ and $c_{n}$ need to be determined from the boundary condition at $r=R$. For a hard disk, it can be shown that

$$
\begin{aligned}
a_{n} & =-\frac{i^{n}}{2 \sqrt{2}} \frac{H_{n}^{1}\left(\tilde{k}^{\prime}\right) H_{n+1}^{2}(\tilde{k})+H_{n}^{2}(\tilde{k}) H_{n+1}^{1}\left(\tilde{k}^{\prime}\right)}{H_{n}^{1}\left(\tilde{k}^{\prime}\right) H_{n+1}^{1}(\tilde{k})+H_{n}^{1}(\tilde{k}) H_{n+1}^{1}\left(\tilde{k}^{\prime}\right)} \\
c_{n} & =\frac{i^{n}}{2 \sqrt{2}} \frac{H_{n}^{1}(\tilde{k}) H_{n+1}^{2}(\tilde{k})-H_{n}^{2}(\tilde{k}) H_{n+1}^{1}(\tilde{k})}{H_{n}^{1}\left(\tilde{k}^{\prime}\right) H_{n+1}^{1}(\tilde{k})+H_{n}^{1}(\tilde{k}) H_{n+1}^{1}\left(\tilde{k}^{\prime}\right)}
\end{aligned}
$$

where $\tilde{k} \equiv k R$ and $\tilde{k}^{\prime} \equiv k^{\prime} R$. Notice that the nonzero probability amplitudes $c_{n}$ lead to outgoing waves with flipped helicity. For reference, if the incident wave is $\Psi_{\text {in,- }}$, then $b_{n}=0$ and $d_{n}=i^{n} / 2 \sqrt{2}$. At the mean time, the roles of $k$ and $k^{\prime}$, as well as the roles of $a_{n}$ and $c_{n}$, have to be interchanged.

A note on the unitary condition: for convenience of discussion, consider an incoming wave with positive helicity and angular momentum $(n+1 / 2) \hbar$ (i.e., the $b_{n}$-wavelet). Because the angular momentum is conserved during the scattering, the electron can only be scattered to $a_{n}$ and $c_{n}$ channels with the same $n$. From particle conservation at large distance, one expects that the probability amplitudes in Eq. (4) should satisfy $\left|a_{n}\right|^{2}+\left(k / k^{\prime}\right)\left|c_{n}\right|^{2}=\left|b_{n}\right|^{2}(=1 / 8)$ for all $n$, which has indeed been confirmed in our numerical calculation.

\section{B. Properties of symmetry}

The system has a mirror symmetry with respect to the $x$-axis. Therefore, by analyzing the Schrodinger equation with $y$ replaced by $-y$, one finds $\Psi\left(\vec{r}^{*}\right)=-\sigma_{y} \Psi(\vec{r})$, where $\vec{r}=(x, y)$, 
and $\vec{r}^{*} \equiv(x,-y)$ is the mirror-reflected point of $\vec{r}$. Such as relation can also be obtained by a space inversion of the (three-dimensional) coordinate, followed by a rotation with respect to the new $y$-axis by 180 degrees. Consequently, for the expectation value of the spin, we have

$$
\left(S_{x}\left(\vec{r}^{*}\right), S_{y}\left(\vec{r}^{*}\right), S_{z}\left(\vec{r}^{*}\right)\right)=\left(-S_{x}(\vec{r}), S_{y}(\vec{r}),-S_{z}(\vec{r})\right)
$$

In a Rashba system, the current density operator is defined as,

$$
\vec{j}=\frac{\hbar}{2 m i}\left(\Psi^{\dagger} \frac{d \Psi}{d \vec{r}}-\frac{d \Psi^{\dagger}}{d \vec{r}} \Psi\right)-\frac{\alpha}{\hbar} \Psi^{\dagger} \vec{\sigma} \times \hat{z} \Psi .
$$

Therefore, the distribution of the expectation value of the current density has the following symmetry:

$$
\left(j_{x}(\bar{r}), j_{y}(\bar{r})\right)=\left(j_{x}(\bar{r}),-j_{y}(\bar{r})\right)
$$

We adopt the generally accepted definition of the spin current density operator, ${ }^{21} \vec{j}_{s}^{\gamma}=$ $\operatorname{Re} \Psi^{\dagger}\left(\sigma^{\gamma} \dot{\vec{r}}\right) \Psi$, whose expectation values have the symmetries,

$$
\begin{aligned}
& \left(j_{x}^{x}\left(\vec{r}^{*}\right), j_{y}^{x}\left(\vec{r}^{*}\right)\right)=\left(-j_{x}^{x}(\vec{r}), j_{y}^{x}(\vec{r})\right) \\
& \left(j_{x}^{y}\left(\vec{r}^{*}\right), j_{y}^{y}\left(\vec{r}^{*}\right)\right)=\left(j_{x}^{y}(\vec{r}),-j_{y}^{y}(\vec{r})\right) \\
& \left(j_{x}^{z}\left(\vec{r}^{*}\right), j_{y}^{z}\left(\vec{r}^{*}\right)\right)=\left(-j_{x}^{z}(\vec{r}), j_{y}^{z}(\vec{r})\right) .
\end{aligned}
$$

These symmetries will be confirmed by the numerical results in Sec. III.

\section{Asymptotic behavior of the scattered wave}

For convenience, the wave function $\sum \Psi_{n}$ can be seperated into an incident plane wave and a scattered wave. At large distance with $k r \gg 1$, the scattered wave has the asymptotic form,

$$
\Psi_{\mathrm{sc}}=\frac{e^{i k r}}{\sqrt{r}}\left(\begin{array}{c}
f_{1}(\phi) \\
f_{2}(\phi)
\end{array}\right)+\frac{e^{i k^{\prime} r}}{\sqrt{r}}\left(\begin{array}{c}
g_{1}(\phi) \\
g_{2}(\phi)
\end{array}\right)
$$

where

$$
\begin{gathered}
\mathbf{f} \equiv\left(\begin{array}{c}
f_{1}(\phi) \\
f_{2}(\phi)
\end{array}\right)=\sqrt{\frac{2}{\pi k}} \sum_{n}\left(a_{n}-b_{n}\right) e^{-i(n+1 / 2) \pi / 2}\left(\begin{array}{c}
e^{i n \phi} \\
-i e^{i(n+1) \phi}
\end{array}\right) \\
\mathbf{g} \equiv\left(\begin{array}{l}
g_{1}(\phi) \\
g_{2}(\phi)
\end{array}\right)=\sqrt{\frac{2}{\pi k^{\prime}}} \sum_{n}\left(c_{n}-d_{n}\right) e^{-i(n+1 / 2) \pi / 2}\left(\begin{array}{c}
e^{i n \phi} \\
i e^{i(n+1) \phi}
\end{array}\right) .
\end{gathered}
$$


It can be shown that $\mathbf{f}^{\dagger} \cdot \mathbf{g}=0$. Also, for the incoming plane wave $\Psi_{\mathrm{in},+}$,

$$
\begin{aligned}
\sum_{i=1}^{2} f_{i}^{*} \vec{\sigma} f_{i} & =|\mathbf{f}|^{2}(\sin \phi,-\cos \phi, 0), \\
\sum_{i=1}^{2} g_{i}^{*} \vec{\sigma} g_{i} & =|\mathbf{g}|^{2}(-\sin \phi, \cos \phi, 0) .
\end{aligned}
$$

Therefore, f-spinor and g-spinor possess spins with opposite directions at large distance. Both spins lie on the plane and are perpendicular to the direction of propagation. One can obtain the same equations for the incoming wave $\Psi_{\text {in,-- }}$, but the signs of the spin expectation values are opposite.

After a straightforward calculation, one can show that the scattered current density at large distance is

$$
\vec{j}_{\mathrm{sc}}=\frac{1}{r}\left(\frac{\hbar k}{m}+\frac{\alpha}{\hbar}\right)|\mathbf{f}|^{2} \hat{r}+\frac{1}{r}\left(\frac{\hbar k^{\prime}}{m}-\frac{\alpha}{\hbar}\right)|\mathbf{g}|^{2} \hat{r},
$$

from which the differential cross-section $\sigma^{\prime}(\phi) \equiv r\left|\vec{j}_{\mathrm{sc}}\right| /\left|\vec{j}_{\text {in }}\right|$ can be calculated. For incoming waves $\Psi_{\text {in, }+}$ and $\Psi_{\text {in,- }}$, the current densities $\left|\vec{j}_{\text {in, },+}\right|$ and $\left|\vec{j}_{\text {in },-}\right|$ are $\hbar k / m+\alpha / \hbar$ and $\hbar k^{\prime} / m-\alpha / \hbar$ respectively. In fact, they are equal in magnitude if the two incident waves have the same energy. Therefore, the differential cross-sections $\sigma_{\eta}$ for incoming waves with helicity $\eta$ are

$$
\begin{aligned}
\sigma_{+}^{\prime} & =\left|\mathbf{f}_{+}\right|^{2}+\left|\mathbf{g}_{+}\right|^{2} \equiv \sigma_{++}^{\prime}+\sigma_{+-}^{\prime} \\
\sigma_{-}^{\prime} & =\left|\mathbf{f}_{-}\right|^{2}+\left|\mathbf{g}_{-}\right|^{2} \equiv \sigma_{-+}^{\prime}+\sigma_{--}^{\prime}
\end{aligned}
$$

where $\mathbf{f}_{\eta}$ is the $\mathbf{f}$-spinor in Eq. (12), but with the coefficients $a_{n}$ and $b_{n}$ suitably chosen for the scattering of $\Psi_{\mathrm{in}, \eta}$, similarly for $\mathbf{g}_{\eta}$ (see the discussion following Eq. (6)). The differen-

tial cross-sections $\sigma_{\eta, \eta}^{\prime}$ and $\sigma_{\eta,-\eta}^{\prime}$ represent helicity-preserved and helicity-flipped scatterings respectively. If the incoming wave is an incoherent mixture of both helicities with fractional populations $P_{\eta}$, then the differential cross-section is simply the weighted average of the two differential cross-sections: $\sigma^{\prime}=P_{+} \sigma_{+}^{\prime}+P_{-} \sigma_{-}^{\prime}$.

\section{DISTRIBUTIONS OF CHARGE AND SPIN}

In the following, we report on the distributions of spin density, charge current density, and spin current density, assuming the wave length of the incident wave $\lambda=R$. We have also studied the cases with a larger $\lambda($ e.g. $\lambda=3 R$ ) and a smaller $\lambda($ e.g. $\lambda=R / 3$ ). These results are not presented since the main difference is the change of scales. In the limit of 


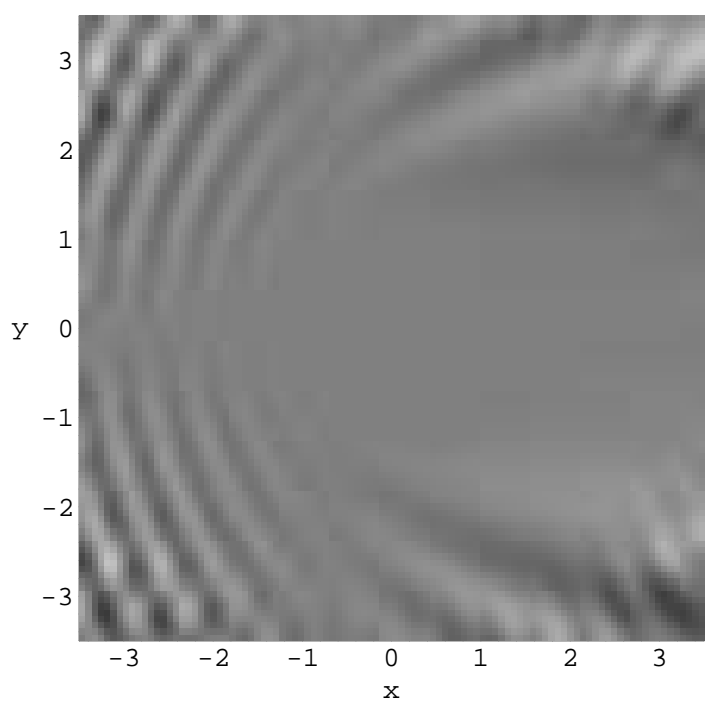

FIG. 1: Distribution of out-of-plane spin $S_{z}$, with $\alpha=(1 / 10) \hbar^{2} k / 2 m$. The incident wave is an equal mixture of both helicities $\eta= \pm$. Lighter (darker) regions represent the regions of spin up (down). The peak intensity in this figure is approximately 0.22 ( 1 for fully polarized).

$\lambda \gg R$, whose scale is more relevant to the case of impurity scattering, only the components with the smallest angular momentum $(n=0$ and -1$)$ need to be considered. From Eq. (66), one finds that both $\left|c_{0}\right|$ and $\left|c_{-1}\right| \rightarrow 0$ as $k R \ll 1$. Therefore, there would be little change of helicity in the long wave length limit.

For comparison with realistic values, we choose $m=0.068 m_{e}$ for electrons in the GaAsAlGaAs heterojunctions. The radius of the disk is fixed at $R=1000 \AA$. The corresponding Fermi energy and electron density for $\lambda_{F}=1000 \AA$ are $2.2 \mathrm{meV}$ and $6.3 \times 10^{10} / \mathrm{cm}^{2}$ respectively, which are typical values. To enhance the visual effect of the spin-orbit coupling, the Rashba energy $\alpha k$ is chosen to be one-tenth of the kinetic energy $\hbar^{2} k^{2} / 2 m$, which requires $\alpha=0.35 \mathrm{~nm}-\mathrm{eV}$, about one order of magnitude larger than the value in GaAs.

The out-of-plane $(z)$ spin component results from a spin-unpolarized incident wave with an equal (incoherent) mixture of both helicities is plotted in Fig. 1, which is antisymmetric with respect to the horizontal $x$-axis: $S_{z}\left(\vec{r}^{*}\right)=-S_{z}(\vec{r})$. Notice that the incident waves with opposite helicities (but the same energy) have different wave vectors $\left(k^{\prime}-k=2 m \alpha / \hbar^{2}\right)$. Therefore, their interference patterns for opposite helicities with spins point at opposite directions are slightly displaced with respect to each other. Because of such a displacement between $\eta=+$ and $\eta=-$, regions with net $z$-spin still exist after partial cancellation. The 


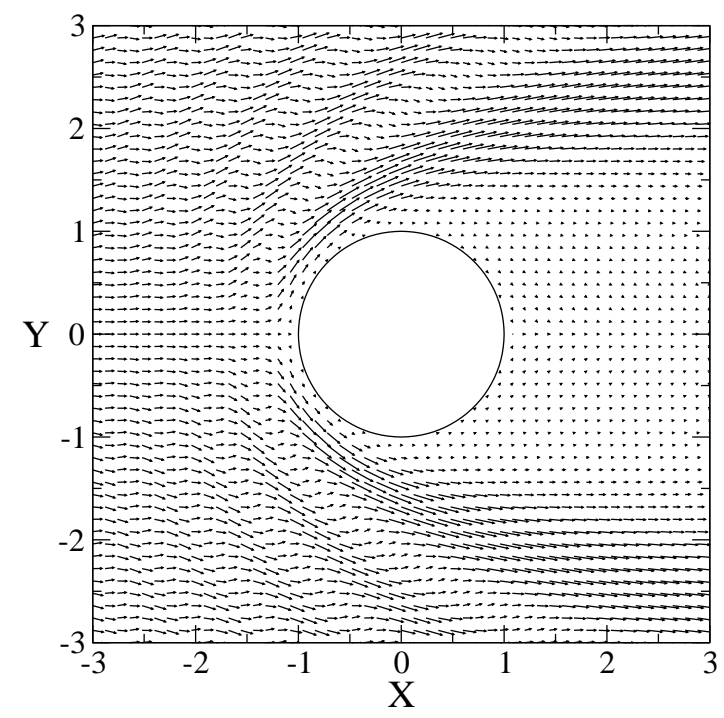

FIG. 2: Distribution of charge current density near the disk. The incident wave is $\Psi_{\mathrm{in},+}$ with wavelength $\lambda=R .\left[\alpha=(1 / 10) \hbar^{2} k / 2 m\right]$

existence of $S_{z}$ relies on the scattered part of the wave function in the near-field region [see Eqs. (11) and (13)] and would decay to zero at large distance.

Unlike the spins in Fig. 1, the distribution of charge current (Fig. 2), as well as the differential cross-section (Fig. 3), which is defined using the charge current densities, are not sensitive to the strength of the Rashba coupling, and look very similar even if the coupling is turned off. Notice that Rashba spin-orbit coupling in fact preserves the helicity. The major cause of the helicity flip is the potential $V(r)$, which is incompatible with the helicity operator. In Fig. 2, the distribution of charge current density from the scattering of $\Psi_{\text {in,+ }}$ shows the expected pattern of the flow around the disk. If the incident wave is a mixed state, then instead of cancellation, the slightly displaced current densities from both helicities will add up.

The differential cross-sections for $\Psi_{\text {in,+ }}$ are shown in Fig. 3. It can be seen that the helicity-preserved scattering $\left(\sigma_{++}^{\prime}\right)$ peaks at the forward direction $(\phi=0,2 \pi)$, while the helicity-flipped scattering $\left(\sigma_{+-}^{\prime}\right)$ peaks at the backward direction $(\phi=\pi)$. At the backward direction, $\sigma_{++}^{\prime}(\pi)=0$. Therefore, the helicity of the electron has to be flipped, but its spin remains conserved. At longer wave length $\lambda=10 R, \sigma_{+-}^{\prime}$ gains more weight and the total differential cross-section $\sigma_{+}^{\prime}=\sigma_{++}^{\prime}+\sigma_{+-}^{\prime}$ becomes more isotropic.

The distributions of spin current density are shown in Fig. 4.22 A prominent feature in the 


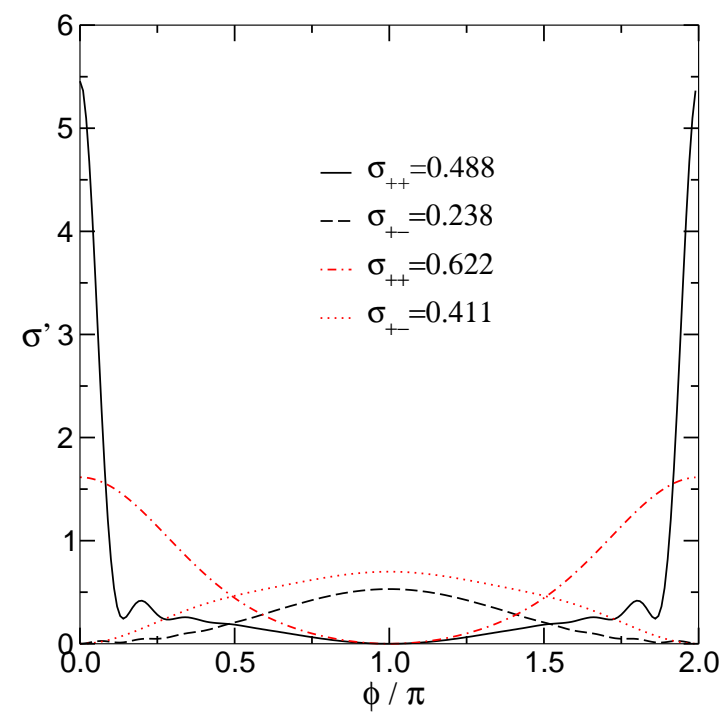

FIG. 3: (Color online) Differential cross-sections (in units of $R$ ) for wave length $\lambda=R$ (solid line for $\sigma_{++}^{\prime}$, dashed line for $\sigma_{+-}^{\prime}$ ) and $\lambda=10 R$ (dash-dotted line for $\sigma_{++}^{\prime}$, dotted line for $\sigma_{+-}^{\prime}$ ). The numbers in the legend are total cross-sections after integration over angle. $\left[\alpha=(1 / 10) \hbar^{2} k / 2 m\right]$

figures is the overall trend for the $\vec{j}_{s}^{y}(\vec{r})$ vectors to point to the left. This counter-intuitive behavior is simply related to the fact that the spin current density is equal to the product of velocity and spin, where the velocity is in the positive $x$-direction and the spin points to the minus $y$-direction for $\Psi_{\mathrm{in},+}$, therefore the scattering $\vec{j}_{s}^{y}$ vectors generically point to the left. On the contrary, for $\Psi_{\text {in,- }}$ with spin points to the positive $y$-direction, the direction of the flow will be reversed. This also explains why the magnitudes of the spin currents $\vec{j}_{s}^{x, z}$ are small in most of the regions, since the original incident current has no $S_{x}$ and $S_{z}$ components.

In Fig. 4, it can be seen that $\vec{j}_{s}^{z}$ oscillates both in amplitude and direction between the curved stripes. An incident wave with opposite helicity would reverse such a flow. Therefore, part of these flows are cancelled if the incident current is not polarized. However, local spin current that oscillates in space still exist, similar to the case of the spin density in Fig. 1. All of the local spin currents $\vec{j}_{s}^{x, y, z}$ would vanish for unpolarized incident electrons once the Rashba coupling is turned off. However, if $\alpha$ is nonzero but the disk is removed (i.e., free space), then there exist spin currents $\vec{j}_{s}^{x}=\alpha / 2 \hat{x}, \vec{j}_{s}^{y}=-\alpha / 2 \hat{y}$, and $\vec{j}_{s}^{z}=\overrightarrow{0}$, which is the background spin current cautioned by Rashba. ${ }^{23}$ It reflects the unsatisfying current status on a proper definition of the spin current. 

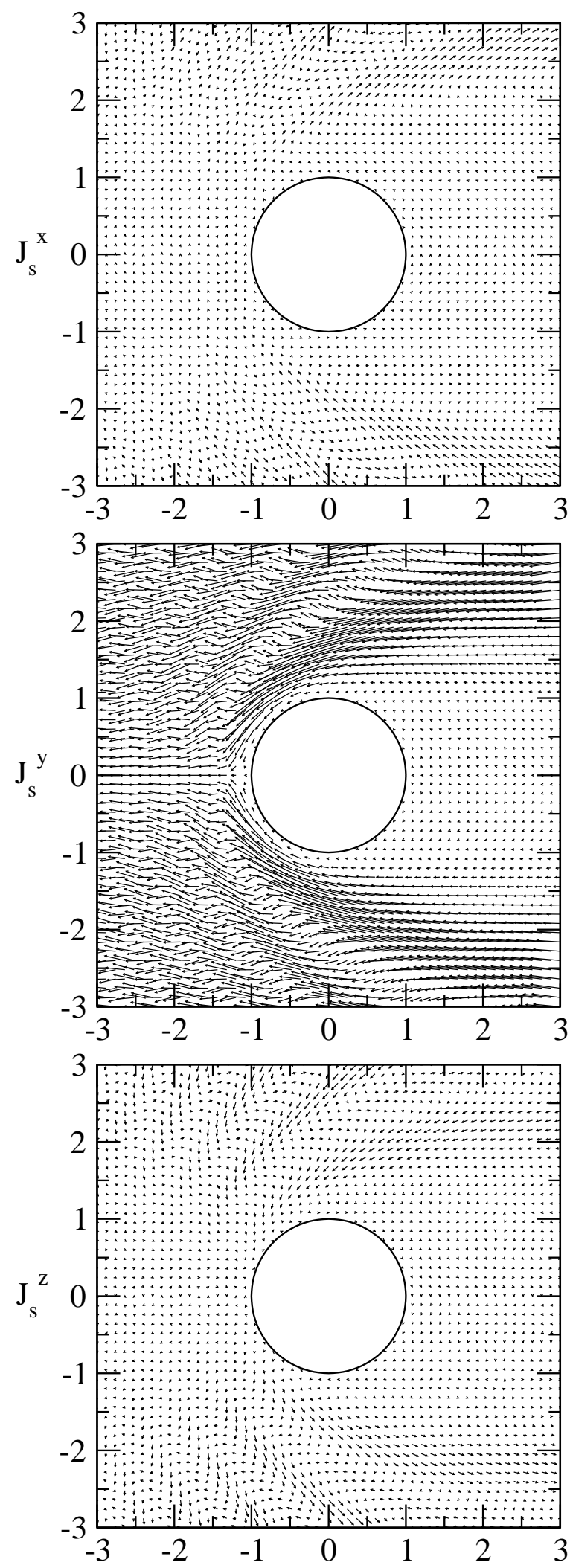

FIG. 4: Distributions of spin current density $\vec{j}_{s}^{x}, \vec{j}_{s}^{y}$, and $\vec{j}_{s}^{z}$. The incident wave has a well-defined helicity $\eta=+$ with $\lambda_{\text {in }}=R \cdot\left[\alpha=(1 / 10) \hbar^{2} k / 2 m\right]$ 
Our system with unilateral current flow seems to be the same as the 2DEG driven by an electric field, $\underline{\underline{4}}$ but there is no global transverse spin current $\vec{j}_{s}^{z}$, no matter the disk is removed or not. This does not contradict the result of the proposed intrinsic spin Hall effect in a clean 2DEG. ${ }^{4}$ The incident charge current flowing to the right can be understood as originating from the slightly unbalanced electrochemical potentials on the two leads far away. In our case, all the electrons are moving along the direction of the potential gradient, instead of

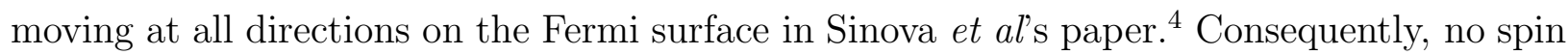
Hall effect is expected if one follows similar semiclassical analysis in Ref. 4 .

\section{CONCLUSION}

In summary, the influence of the spin-orbit coupling on the scattering of $2 \mathrm{D}$ electrons from a hard disk is studied. Such a simple setup offers us a good opportunity to investigate the properties of spin and spin current in details. We focus our attention on the near-field regime, where the scattered wave is comparable to the incident wave, and appreciable outof-plane spins can be found. This work offers us a clear understanding of the microscopic dynamics around the mesoscopic disk, and could serve as a basis for future works considering a threaded magnetic flux in the disk, or a hybrid device involving a disk as a component.

\section{Acknowledgments}

The authors acknowledge the support from the National Science Council in Taiwan. M.C.C. thanks M.F. Yang for helpful discussions.

* corresponding e-mail address changmc@phy.ntnu.edu.tw

1 Quantum Theory of Solids, by C. Kittel, Wiley \& Sons, 1963.

2 J.E. Hirsch, Phys. Rev. Lett. 83, 1834 (1999).

3 S. Murakami, N. Nagaosa, and S. C. Zhang, Science 301, 1348 (2003); S. Murakami, cond-mat/0405003.

4 Jairo Sinova, Dimitrie Culcer, Q. Niu, N. A. Sinitsyn, T. Jungwirth, and A. H. MacDonald, Phys. Rev. Lett. 92, 126603 (2004). 
5 Igor Zutic, Jaroslav Fabian, S. Das Sarma, Rev. Mod. Phys. 76, 323-410 (2004); W. Zawadzki and P. Pfeffer, Semicond. Sci. Technol. 19, R1 (2004).

6 Optical Orientation, edited by F. Meier and B.P. Zakharchenya, North-Holland, 1984.

7 E. I. Rashba, Sov. Phys. Solid State 2, 1224 (1960); Y. A. Bychkov and E. I. Rashba, JETP Lett. 39, 78 (1984).

8 For example, the current modulator proposed by S. Datta and B. Das, Appl. Phys. Lett. 56, 665 (1990); Marco G. Pala, Michele Governale, Jurgen Konig, Ulrich Zülicke, and Giuseppe Iannaccone, Phys. Rev. B 69, 045304 (2004).

9 M. Governale, U. Zülicke, Phys. Rev. B 66, 073311 (2002); M. Governale, U. Zülicke, cond-mat/0407036.

10 J. Splettstoesser, M. Governale, U. Zülicke, Phys. Rev. B 68, 165341 (2003); S.Q. Shen, Z.J. Li, and Z. Ma, App. Phys. Lett. 84, 996 (2004); S. Souma and B.K. Nikolić, Phys. Rev. B 70, 195346 (2004); G.S. Lozanno and M.J. Sánchez, cond-mat/0505394.

11 B.I. Halperin, Ady Stern, Yuval Oreg, J.N.H.J. Cremers, J.A. Folk, and C.M. Marcus, Phys. Rev. Lett. 86, 2106 (2001); W.H Kuan, C.S. Tang, W. Xu, cond-mat/0403098; E. Tsitsishvili, G.S. Lozano, and A.O. Gogolin, Phys. Rev. B 70, 115316 (2004).

12 A short but insightful review on spintronics can be found in M.I. Dyakonov, cond-mat/0401369.

13 A.A. Kiselev and K.W. Kim, Appl. Phys. Lett. 78, 775 (2001).

14 V.M. Ramaglia, D. Bercioux, V. Cataudella, G. De Filippis, C.A. Perroni, and F. Ventriglia Eur. Phys. J. B 36, 365 (2003).

15 V. M. Ramaglia, D. Bercioux,V. Cataudella, G. De Filippis and C. A. Perroni, J. Phys.: Condens. Matter 16, 9143 (2004).

16 M.P. Trushin and A.L. Chudnovskiy, cond-mat/0505104.

17 Gonzalo Usaj and C. A. Balseiro, Phys. Rev. B 70, 041301 (R) (2004); L.P. Rokhinson, Y.B. Lyanda-Geller, L.N. Pfeiffer, K.W. West, Phys. Rev. Lett. 93, 146601 (2004).

18 R. Ionicioiu and I. D'Amico, Phys. Rev. B 67, 041307(R) (2003).

19 In the absence of spin-orbit coupling, the study of two-dimensioanl electron scattering by a disk can be found in I.R. Lapidus, Am. J. Phys. 50, 45 (1982); P.A. Maurone and C. Shiomos, ibid. 51 (1983); I.R. Lapidus, ibid. 54 (1986).

20 This report is abridged from Jr-Yu Yeh's master thesis (July, 2005, National Taiwan Normal Univ.), in which the distributions of charge, spin, charge current, and spin current are studied 
using various ratios of $\lambda / R$.

21 See, for example, S.I. Erlingsson, J. Schliemann, D. Loss, Phys. Rev. B 71, 35319 (2005); E.I. Rashba, cond-mat/0404723.

22 We have also plotted the same distributions using the spin current recently proposed by Ping Zhang, Junren Shi, Di Xiao, and Qian Niu, cond-mat/0503505. For $\vec{j}_{s}^{x}$ and $\vec{j}_{s}^{y}$, the patterns of the variation look similar to the ones shown in Fig. 4; for $\vec{j}_{s}^{z}$, the stripes on the left of the disk are enhanced and more visible.

23 E.I. Rashba, cond-mat/0408119, invited talk at the 3rd Intern. Conf. on Physics and Applications of Spin-Related Phenomena in Semiconductors, Santa Barbara (CA), July 21-23. 